\title{
Major Groove
}

National Cancer Institute

\section{Source}

National Cancer Institute. Major Groove. NCI Thesaurus. Code C13743.

One of the two types of grooves found in a right-handed double helix, running between the sugar phosphate backbones of each strand. 